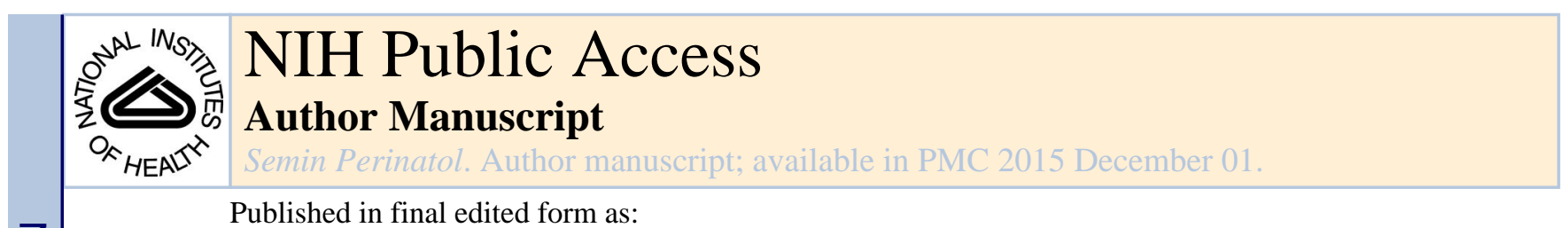

Published in final edited form as:

Semin Perinatol. 2014 December ; 38(8): 487-495. doi:10.1053/j.semperi.2014.08.016.

\title{
Pharmacological Management of Hypertension in Pregnancy
}

\author{
Thomas R. Easterling, MD \\ Professor, Department of Obstetrics and Gynecology, University of Washington, Seattle, WA \\ 98195
}

Thomas R. Easterling: easter@u.washington.edu

\begin{abstract}
Hypertension in pregnancy remains a significant public health problem. Pharmacological management of blood pressure in pregnancy is impacted by changes in maternal drug disposition and by the pharmacodynamic effects of specific agents. This paper will review the impact of pregnancy on pathways of drug elimination and the associated clinical implications, the pharmacodynamic effects of specific drugs and classes of drugs in pregnancy, and the data to date on the impact of antihypertensive therapy on mothers and their fetuses.
\end{abstract}

\section{Introduction}

Hypertension in pregnancy remains a significant public health problem. Preeclampsia, chronic hypertension, and severe gestational hypertension, while subject to different diagnostic criteria, each contributes to maternal and perinatal morbidity and mortality. Hypertensive pregnant women are at risk for cerebrovascular accident, cerebral edema, hepatic rupture, renal failure, heart failure and death. Hypertension diagnosed in pregnancy identifies women at risk for subsequent cardiovascular disease when not pregnant. The fetuses of hypertensive women are at risk for complications of preterm birth after delivery for maternal indications, intrauterine growth restriction and stillbirth. The risk for the severest of outcomes such as maternal mortality and cerebral injury is moderated through prenatal care. Indicated early delivery protects the mother and the neonate from stillbirth often at the cost of preterm delivery and its associated complications.

A number of "single molecule adjunctive therapies" have been suggested for the prevention of preeclampsia with the potential for improving maternal and perinatal outcomes. Large trials of treatment of high and low risk women with aspirin, calcium and antioxidants have not supported benefit. Meta-analyses, including smaller trials with greater variability, have continued to support some potential benefit for aspirin - but with relatively limited impact.

(c) 2014 Elsevier Inc. All rights reserved.

Conflict of Interest/Disclosure - The author reports no proprietary or commercial interest in any product mentioned or concept discussed in this article.

Publisher's Disclaimer: This is a PDF file of an unedited manuscript that has been accepted for publication. As a service to our customers we are providing this early version of the manuscript. The manuscript will undergo copyediting, typesetting, and review of the resulting proof before it is published in its final citable form. Please note that during the production process errors may be discovered which could affect the content, and all legal disclaimers that apply to the journal pertain. 
Outside pregnancy, hypertension is clearly associated with adverse outcomes and treatment has been demonstrated to improve outcomes. Treatment of hypertension in pregnancy remains controversial in part due to assumptions that high blood pressure itself is not "in the pathway" of adverse outcomes. Some advocate only treating severe hypertension (>160/110 $\mathrm{mmHg}$ ) and then treating aggressively with parenteral medications. In the absence of conflicting data, others argue that pregnant women should be treated as one would a woman who is not pregnant. Given the severity of associated maternal complications and the short time frame of disease progression, others argue that women at high risk for adverse outcomes should be treated aggressively by high risk JNC-7 standards. ${ }^{1}$ Each of these positions seeks to balance the risk of adverse maternal outcome and of preterm delivery against the potential risk of treatment to the fetus - in particular the impact on fetal growth. Statements of opinion have been published with acknowledgement that clear supporting data is lacking. Recommendations and opinions remain controversial. That said, the failure to adequately treat hypertension has been cited by the Joint Commission ${ }^{2}$ as a major cause of preventable maternal mortality in the United States.

The American College of Obstetricians and Gynecologists has recently completed a review of the management of hypertension in pregnancy by a task force of experts in the field. ${ }^{3}$ The task force was able to make six recommendations where the quality of evidence was "High" and the recommendation was "Strong." Of five recommendations pertaining to pharmacological management, two supported the use of antenatal steroids in hypertensive women to improve pulmonary function in babies born prematurely. Two supported the use of magnesium sulfate in women with severe hypertension. One recommended against the use of antioxidant supplementation with vitamin $\mathrm{C}$ and $\mathrm{E}$. An additional nineteen recommendations were made where the quality of evidence was "Moderate" and the strength of recommendation was "Strong." Eight of the nineteen pertained to pharmacological management. Two supported the use of antenatal steroids and two supported the use of magnesium sulfate. Two recommended that systolic blood pressure $\geq 160 \mathrm{mmHg}$ and diastolic blood pressure $\geq 105 \mathrm{mmHg}$ should be treated. One recommended labetalol, nifedipine and methyldopa for initial management of hypertension in pregnancy. One recommendation cautioned against the use of angiotensin-converting enzyme inhibitors, angiotensin receptor blockers, renin inhibitors and mineralocorticoid receptor antagonists in pregnancy. An additional thirty-one recommendations were made where the quality of evidence was "Low" to Moderate and the strength of recommendation was "Qualified." The report of the Task Force on Hypertension in Pregnancy highlights the need for research in the field: "...there are few recommendations that can be classified as "strong" because there are huge gaps in the evidence that guides therapy. These knowledge gaps form the basis for future research recommendations to guide therapy."3

A variety of antihypertensive medications have been used in pregnancy. Table 1 lists those commonly used and summarizes their pathways of drug disposition, the impact of pregnancy on these pathways when known, the primary mechanism of action and the primary and secondary hemodynamic changes. This paper will review the pharmacokinetics and pharmacodynamics of antihypertensive drugs in pregnancy and then briefly summarize information to date on efficacy. 


\section{Pharmacokinetics of Antihypertensive Agents in Pregnancy}

Physiological adaptations to pregnancy directly impact drug disposition. Increased cardiac output in pregnancy is associated with increased renal blood flow and hepatic blood flow. Increased renal blood flow directly increases glomerular filtration and the clearance of many drugs. Increased activity of renal tubular transporters such as p-glycoprotein and organic ion transporters will increase renal secretion clearance in excess of that due to increased GFR alone. When present in the placenta, transporters may limit transport to the fetus. Increased hepatic blood flow will increase the first pass metabolic clearance of drugs with high extraction ratios. Induction of cytochrome p-450 enzymes (CYP-3A, CYP-2D6) and conjugation activity will increase metabolic clearance of other drugs.

Atenolol and metoprolol are selective $\beta_{1}$-blockers with comparable antihypertensive properties but different routes of drug disposition. The pharmacokinetics of atenolol in pregnancy has been studied in 20 women treated for hypertension in pregnancy. ${ }^{4}$ The subjects were relatively obese with an average weight prior to pregnancy of $112 \pm 26.5 \mathrm{~kg}$ and an average creatinine clearance of $224 \mathrm{~mL} / \mathrm{min}$ pregnancy which could affect generalization to all pregnant women. They were studied in the $2^{\text {nd }}$ and $3^{\text {rd }}$ trimesters and 3 months postpartum. As would be expected the renal clearance of atenolol was increased in the $2^{\text {nd }}(14.7 \pm 3.6 \mathrm{~L} / \mathrm{hr})$, and $3^{\text {rd }}$ trimesters $(14.6 \pm 3.5 \mathrm{~L} / \mathrm{hr})$ compared to postpartum $(11.1 \pm 3.5 \mathrm{~L} / \mathrm{hr}), \mathrm{P}<0.001 .{ }^{4}$ Renal clearance was strongly correlated with creatinine clearance $(\mathrm{r}=0.665) .{ }^{4}$ Despite sufficient power to detect a $28 \%$ change, no difference in the apparent oral clearance could be demonstrated between the $2^{\text {nd }}(31.1 \pm 8.5 \mathrm{~L} / \mathrm{h})$ or $3^{\text {rd }}$ trimesters $(32.7 \pm 9.6 \mathrm{~L} / \mathrm{h})$ and postpartum values $(29.2 \pm 13.2 \mathrm{~L} / \mathrm{h}) .{ }^{4}$ Apparent oral clearance is the total clearance of an orally administered drug assuming that the entire dose has been absorbed and then cleared. It therefore reflects not only drug disposition but also bioavailability. The high variability in apparent oral clearance observed in pregnancy probably reflects variability in absorption and bioavailability. Given modest absolute differences in apparent clearance and high variability coupled with increased adrenergic tone in pregnancy, changes in atenolol dose in a pregnant woman are difficult to estimate. Fortunately, the dose effect can be evaluated based on maternal heart rate and blood pressure response. A significant difference in half-life in pregnant compared to non-pregnant women (4.8 \pm 0.6 vs. $5.7 \pm 1.2$, $\mathrm{P}=0.009){ }^{4}$ probably justifies a change from once a day dosing to twice a day dosing though this dosing strategy has not been directly tested.

Metoprolol is a substrate of CYP2D6. CYP2D6 is cytochrome p450 enzyme generally found not to be inducible by pharmacological agents. Substantial individual variability in CYP2D6 activity due to enzyme polymorphisms leads to the characterization of individuals as poor metabolizers, heterozygous extensive metabolizers and ultra-rapid metabolizers. ${ }^{5}$ Using dextromethorphan as a probe for CYP2D6 activity, a marked increased in activity in pregnancy has been described among heterozygous extensive metabolizers and extensive metabolizers. ${ }^{6}$ In a study of 8 pregnant women, peak plasma concentrations of metoprolol were found to be 4 -fold lower in the $3^{\text {rd }}$ trimester compared to the postpartum period. ${ }^{7}$ Currently, larger studies are underway that will be able to characterize the changes in disposition in the context of specific enzyme polymorphisms. Many pregnant women treated with metoprolol will require substantial increases in dose to achieve an equivalent effect in 
pregnancy. An increase in total dosage is usually accompanied by a need for a decreased dosing interval. Loss of heart rate control is usually a sign of an increase in drug clearance often apparent in the late $1^{\text {st }}$ or early $2^{\text {nd }}$ trimesters. The differential response among women with enzyme polymorphisms ranging from slow metabolizers to extensive metabolizers makes prediction of the extent of need for change in drug dosage difficult to anticipate. Based on observed changes in maternal serum concentrations, ${ }^{7}$ some patients may need 4fold increases in dosage - frequently to levels beyond the experience of many providers. In some cases, substituting atenolol for metoprolol may be chosen rather than resorting to large doses of metoprolol and requirements of dosing four times per day. Propranolol and carvedilol are also substrates for CYP2D6. While specific PK studies are not available in pregnancy, the results for metoprolol can probably be clinically extrapolated to these drugs.

Labetalol is a nonselective $\beta$-blocker and an $\alpha_{1}$-blocker that is widely recommended for management of hypertension in pregnancy. Disposition is mediated by glucuronidation via UDP-glucuronosyltransferase $1 \mathrm{~A}$ and $2 \mathrm{~B} 7$. Early reports suggest a total terminal elimination half-life of $1.7 \pm 0.27$ hours, substantially shorter than that reported outside pregnancy, approximately 6-8 hours. ${ }^{8}$ Labetalol is a chiral drug with two diastereomeric pairs of racemates. (RR)-labetalol is a nonselective $\beta$-blocker; (SR)-labetalol is a $a_{1}$-blocker. ((SS)labetalol and (RS)-labetalol have little activity.) ${ }^{9}$ When administered intravenously the pharmacokinetics are not stereoselective; the $\beta$-blocking isomer (RR)-labetalol and the $\alpha_{1^{-}}$ blocking isomer (SR)-labetalol are cleared at the same rate. ${ }^{9}$ When administered orally in pregnancy the apparent oral clearance of (RR)-labetalol (4.4; CI 36-7.4 L/h/kg) is higher than for (SR)-labetalol (2.9; CI 2.0-4.9 L/h/kg). ${ }^{9}$ The AUC for (RR)-labetalol (45.6; CI $40.3-74.4 \mathrm{ng} \bullet \mathrm{h} / \mathrm{mL}$ ) is roughly half that for (SR)-labetalol (84.2; CI $63.8-119 \mathrm{ng} \bullet \mathrm{h} / \mathrm{mL}) .{ }^{9}$ Stereoselective glucuronidation of orally administered drug results in more rapid clearance of the $\beta$-active isomer compared to the $a$-active isomer limiting the impact of oral labetalol on maternal heart rate - particularly at lower doses. Clinically, the pharmacokinetics of labetalol can be used to inform dosing in pregnancy. With a half-life $<2.0$ hours, a standard 12 hour dosing interval (6 half-lives) would not be expected to be effective. Dosing at 6 or 8 -hour intervals would be more appropriate. The pharmacodynamic effect of oral labetalol will be very different from that of IV labetalol. When administered orally, labetalol will act with greater a-effect than $\beta$-effect. If heart rate control is required, a substantial higher dose of labetalol may be required. In some cases, labetalol will not control heart rate and a pure $\beta$ blocker will be required.

Clonidine and methyldopa are central acting $a_{2}$ agonists. Their antihypertensive effects are achieved by stimulating $\mathrm{a}_{2}$ receptors in the brainstem thereby decreasing central adrenergic output. To have effect they must enter the central nervous system. Clonidine enters the CNS more rapidly than methyldopa and therefore has a quicker onset of action. Clonidine has a more benign profile of side effects. In non-pregnant patients the clonidine disposition is primarily renal with $60 \%$ eliminated unchanged in the urine. In pregnancy apparent oral clearance of clonidine is increased $(440 \pm 168 \mathrm{ml} / \mathrm{min})$ compared to non-pregnant subjects $(245 \pm 72 \mathrm{ml} / \mathrm{min}), \mathrm{P}<0.0001 .{ }^{10}$ Despite an expected increase in creatinine clearance in pregnancy, clonidine renal clearance $(153 \pm 67 \mathrm{ml} / \mathrm{min})$ is not increased compared to nonpregnant subjects $(183 \pm 55 \mathrm{ml} / \mathrm{min}), \mathrm{p}=0.3 .{ }^{10}$ In addition, only $36 \pm 11 \%$ of clonidine was 
excreted unchanged in the urine compared with $59 \pm 18 \%$ in non-pregnant subjects, $\mathrm{P}<0.0001 .{ }^{10}$ The markedly increased apparent oral clearance in pregnancy juxtaposed to no increase in renal filtration clearance suggests an alternative predominant pathway of drug disposition in pregnancy. Using human liver microsomes, in vitro clearance of clonidine was found to be inhibited by furafylline, (a specific inhibitor of CYP2D6), and not inhibited by ketoconazole, (a specific inhibitor of CYP3A). ${ }^{11}$ When subjects were genotyped for CYP2D6 phenotype, extensive metabolizers and poor metabolizers clearly segregated according to their non-renal clonidine clearance. ${ }^{11}$ Changes in drug metabolism in pregnancy, induction of CYP2D6, changed the fundamental pathway of drug disposition of clonidine. While this finding is to date unique to clonidine, other drugs, where the pharmacokinetics have not been characterized in pregnancy, may be subject to the same effect. Clinically, the increased apparent oral clearance of clonidine in pregnancy suggests a benefit to shorter dosing frequency than twice a day commonly used outside pregnancy. Dosing every 8 hours seems to provide continuous coverage. As a substrate of CYP2D6, one should expect variability in clearance given common polymorphisms of extensive and slow metabolism.

Nifedipine is a calcium channel blocker and, like most calcium channel blockers, is metabolized by CYP3A. Midazolam is a selective probe for CYP3A activity. In a study of pregnant women who served as their own controls postpartum, the apparent oral clearance of midazolam in pregnancy $(4.2 \pm 1.8 \mathrm{~L} / \mathrm{min})$ was increased compared to apparent oral clearance when not pregnant $(2.0 \pm 0.6 \mathrm{~L} / \mathrm{min}), \mathrm{p}=0.002 .{ }^{12}$ Clearance increased by $108 \%$ suggesting a marked increase in CYP3A activity in pregnancy. ${ }^{12}$ In 15 women studied with hypertension in pregnancy, apparent oral clearance $(2.0 \pm 0.8 \mathrm{~L} / \mathrm{hr} / \mathrm{kg})$ was increased compared to non-pregnant subjects $(0.49 \pm 0.09 \mathrm{~L} / \mathrm{hr} / \mathrm{kg}) .{ }^{13}$ In 8 women studied immediately postpartum, apparent oral clearance was increased $(3.3 \pm 1.3 \mathrm{~L} / \mathrm{hr} / \mathrm{kg})$ compared to pregnant women in the $3^{\text {rd }}$ trimester and non-pregnant controls. ${ }^{14}$ The impact of increased metabolism due to CYP3A remains important in the immediate postpartum period. Similar to CYP2D6 there are polymorphisms of CYP3A that impact drug disposition. The apparent oral clearance for pregnant women with high CYP3A5 expression $(232 \pm 37.8 \mathrm{l} / \mathrm{h})$ was substantially greater than that for those with low expression of CYP3A5 variants *3, *6, or*7 (85.6 \pm 45.0$), \mathrm{p}<0.0001 .{ }^{15}$ Clinically, immediate release nifedipine is dosed every 8 hours. The increased clearance described in pregnancy would suggest a more frequent dosing interval (every 6 hours). Extended release nifedipine may require a dosing interval of every 8 hours. The presence of individuals with low expression of CYP3A5 indicates that some women may be very sensitive to lower doses of nifedipine. Given a potential for hypotension associated with a pure vasodilator, care should be exercised when initiating nifedipine for the treatment of hypertension.

Pharmacokinetic studies of hydralazine and furosemide in pregnancy are not available.

\section{Pharmacodynamics of Antihypertensive Agents in Pregnancy}

The mechanisms of pharmacodynamic action of antihypertensive agents can be characterized by Ohm's Law where pressure is a function of flow and resistance. Blood pressure can be lowered by reducing cardiac output or by reducing vascular resistance. 
Cardiac output, the product of heart rate and stroke volume, can be lowered by reducing either component variable. As a single variable is manipulated pharmacologically, compensatory responses can be expected. A reduction in heart rate to achieve a lower cardiac output and blood pressure will usually be accompanied by an increase in stroke volume and vascular resistance. Treatment with a pure vasodilator such as hydralazine will result in an increase in stroke volume and heart rate as blood pressure is reduced. Figure 1 illustrates these concepts. The structure of this diagram is derived from Ohm's Law where $\mathrm{MAP}=(\mathrm{TPR} / 80) \cdot \mathrm{CO},(\mathrm{MAP}-\mathrm{mean}$ arterial pressure, TPR-total peripheral resistance, $\mathrm{CO}$ cardiac output). This is a linear equation where MAP is plotted on the y-axis and $\mathrm{CO}$ is plotted on the $\mathrm{x}$-axis. TPR/80 therefore represents the slope of the relationship between MAP and CO resulting in a set of isometric lines that will converge at the origin. These isometric lines describe the locus of points with the same vascular resistance. Changes in blood pressure that are the result of pure vasodilation will be represented by vectors that run perpendicular to lines of resistance. With a reduction of blood pressure due to vasodilation, cardiac output increases. Changes in blood pressure due to a reduction of cardiac output alone will be represented by vectors of change that run parallel to lines of resistance. A number of drugs commonly used in pregnancy have been studied with noninvasive measurement of maternal hemodynamics.

Hydralazine lowers blood pressure by directly relaxing vascular smooth muscle through alterations in intracellular calcium metabolism. In Figure 1, the vector labeled "hydralazine" demonstrates the hemodynamic changes observed in 10 pregnant hypertensive women with elevated vascular resistance treated with oral hydralazine. ${ }^{16}$ The observed vector of effect runs perpendicular to lines of resistance as would be expected with the assumed mechanism of action. A modest reduction in mean arterial pressure, $(-6.9 \pm 7.0 \mathrm{mmHg}, 6 \%$ below baseline), is accompanied by a substantial increase in cardiac output $(2.0 \pm 1.3 \mathrm{~L} / \mathrm{min}, 38 \%$ above baseline). ${ }^{16}$ The impact on cardiac output, not generally measured in clinical practice, is proportionally greater than the observed change in blood pressure. Similar data has not been generated for nifedipine and other calcium channel blockers. To the extent that the assumed mechanism of action is true, vasodilation, we would expect similar vectors of action.

Atenolol lowers blood pressure through its action on $\beta$-receptors and a reduction in heart rate. In Figure 1, the vector labeled "atenolol" demonstrates the hemodynamic changes observed in 9 hypertensive pregnant women with elevated cardiac output treated with oral atenolol. ${ }^{16}$ The observed vector of change runs roughly parallel to lines of resistance but with some deviation towards a higher resistance. The increase in vascular resistance varied between patients and probably represents a compensatory response to maintain an elevated blood pressure. Similar data does not exist for metoprolol or propranolol. To the extent that the assumed mechanism of action is observed, $\beta$-blockade, we would expect similar vectors of action.

Clonidine lowers blood pressure through stimulation of $a_{2}$ adrenergic receptors in the central nervous system lowering central adrenergic output. In Figure 1, the vector labeled "clonidine" demonstrates the hemodynamic changes observed in 66 hypertensive pregnant women with elevated vascular resistance treated with oral clonidine. ${ }^{17}$ The observed vector 
of change is intermediate between one of pure vasodilation and one of pure reduction in cardiac output. This intermediate vector is the result of substantial heterogeneity of response by individual subjects. Of the 66 women studied, $34,(52 \%)$ exhibited a response consistent with vasodilation; $22,(33 \%)$ exhibited a response similar to that of a $\beta$-blocker with a reduction of heart rate and cardiac output. ${ }^{17}$ Given that baseline central adrenergic output regulates both cardiac (stimulation of heart rate) and renal (stimulation of renin secretion) functions, therapeutic effects of central adrenergic stimulation would be expected to vary between individuals.. This is presumably the basis for differences in the pharmacodynamics effects of clonidine. To the extent that methyldopa has a similar mechanism of action, we would expect similar vectors of action.

Furosemide is a diuretic that blocks the reabsorption of sodium at the loop of Henle. In Figure 1, the vector labeled "furosemide" demonstrates the hemodynamic changes observed in 21 hypertensive pregnant women with elevated cardiac output treated with oral furosemide. ${ }^{18}$ The observed vector of change demonstrates a reduction in cardiac output but with a more marked deviation towards a higher resistance when compared to the atenolol vector. Treatment did not change mean arterial pressure, but reduced stroke volume $(-17$ $\mathrm{mL}-12 \%)$ and cardiac output $(-1.2 \mathrm{~L} / \mathrm{min}-12 \%) .{ }^{18}$ Vascular resistance increased 101 dyne $\bullet$ sec $\bullet \mathrm{cm}^{-5}(+14 \%)$, probably representing a compensatory response to maintain an elevated blood pressure. ${ }^{18}$ Similar data does not exist for hydrochlorothiazide. To the extent that the assumed mechanism of action is true, diuresis, we would expect similar vectors of action.

Labetalol achieves a reduction in blood pressure through its antagonistic effect on of $a_{1}$ and $\beta$ cardiovascular receptors. As a vasodilator through a-antagonism, one would expect a reduction in vascular resistance accompanied by an increase in cardiac output as is seen with hydralazine. As a $\beta$-blocker, one would expect a reduction in $\mathrm{CO}$ and $\mathrm{HR}$ as seen with pure $\beta$-blockers such as atenolol. If the $\alpha$ and $\beta$ effects of labetalol are balanced, one would expect a reduction in vascular resistance with little change in cardiac output or heart rate. This would be seen as a vertical vector in Figure 1 compared to the vectors of pure vasodilators (eg. hydralazine) and pure $\beta$-blockers, (eg. atenolol). Given what appears to be differential metabolism of specific labetalol isomers in pregnancy, one might expect a loss of balanced effect with less $\beta$-antagonism or substantial variability in pharmacodynamic effect between subjects. Higher doses of labetalol may serve to achieve a greater $\beta$-effect. The pharmacodynamic effects of oral labetalol in pregnancy have not been explored.

Maternal hemodynamic information is not commonly available in routine practice to guide treatment. However, understanding the principles of pharmacodynamic effect can be used to inform therapy. This is particularly true when more than a single agent is needed to control blood pressure. For example, most patients with a mean arterial pressure of $130 \mathrm{mmHg}$ (Figure 1) will have a mixed hemodynamics profile of elevated resistance and cardiac output. The treatment effect of combination drug therapy can be predicted by adding individual drug vectors, "head to tail." Use of complimentary agents can result in more efficient blood pressure control. 
The hemodynamic changes associated with hypertension in preeclampsia are heterogeneous and dynamic over the time course of pregnancy. Prior to the onset of clinically apparent disease, the cardiac output of nulliparous women destined to develop preeclampsia is elevated above that of women who do not develop preeclampsia. ${ }^{19,20}$ With the onset of clinical disease maternal hemodynamics become much more varied characterized by acute increases in vascular resistance in some women and a persistence of high cardiac output in others. ${ }^{20}$ Individualizing therapy to protect maternal interests while simultaneously optimizing conditions to support fetal growth requires an understanding of the pharmacodynamics effects described above in the context of the potential hemodynamic characteristics of individual pregnancies.

\section{Impact of Antihypertensive Therapy on Maternal and Fetal Outcomes}

Clinical trials of treatment of hypertension in pregnancy are limited in scope and size.

Approximately 28 trials including 3200 women exposed to a wide range of treatments can be found. In contrast, conclusions drawn from clinical trials in The JNC-7 Report for nonpregnant care was based on 19 studies with 58,872 enrolled subjects. ${ }^{1}$ In the absence of definitive clinical trials, information derived from meta analysis of existing small trials and from administrative data bases can be used to examine pregnancy outcomes in the context of exposure to antihypertensive agents. Each of these approaches has fundamental limitations and, therefore, any conclusions must be viewed with caution. Nevertheless, the results of these analyses can be used in the context of good clinical judgment to guide care and should be used to design more rigorous trials.

Women treated with antihypertensive agents in pregnancy have more adverse outcomes in pregnancy. Orbach et al, in a study of 433 treated women, reported that exposure to antihypertensive agents was associated with increase rates of growth restriction [OR 4.37; CI 3.00-6.36] and preterm delivery [OR 3.69; CI 2.90-4.69] compared to non-hypertensive pregnant women. ${ }^{21} \mathrm{~A}$ similar risk was reported for untreated hypertensive women compared to normotensive women. In a study of 2459 women exposed to pure $\beta$-blockers or labetalol compared to women not prescribed medication, Meidahl also found an increased risk of SGA [aOR 1.98; CI 1.75-2.23] preterm birth [aOR 2.26; CI 2.03-2.52] and perinatal mortality [aOR 1.89 ; CI 1.25-2.84]. ${ }^{22}$ In each, the authors conclude that the risk described is likely due to the underlying disease process rather than treatment. Clearly treatment of hypertension does not result in a complete normalization of risk profile.

Administrative data-bases have also been used to evaluate outcomes by specific drug exposure. Lydakis et al, in a study of 221 treated pregnancies, reported increased rates of SGA in women treated with multiple drug regimens (45.6\%) and atenolol (33.3\%) and women treated with non-atenolol monotherapy (26.4\%) compared to untreated women $(20.9 \%) .^{23}$ Their analysis suggested that early treatment might have a greater impact on fetal growth. Confounding the results and conclusions was a higher incidence of preterm delivery in the atenolol and multiple drug groups suggesting more clinically significant disease in these groups where slower growth might be expected. ${ }^{23}$ Similarly, pregnancies requiring treatment earlier in pregnancy might represent more significant disease. ${ }^{23}$ In contrast, Orbach reported a higher incidence of preeclampsia (27\% vs. $17 \%)$ and preterm delivery 
(17\% vs. $9 \%$ ) in pregnant women treated with methyldopa compared to $\beta$-blockers. ${ }^{21}$

Associated with the observed increased rate of delivery at term in the atenolol cohort was an increased rate of SGA $(5.6 \%)$ compared to methyldopa (2.4\%) but comparable rates of IUGR, $(4.32 \%$ vs. $5.19 \%){ }^{21}$ They did not find differences based on early therapy as were reported by Lydakis. ${ }^{21}$ Meidahl also stratified results based on exposure to pure $\beta$-blockers compared to labetolol. ${ }^{22}$ The observed risk of SGA associated with labetalol [aOR 2.12; 1.72-2.37] was comparable for that associated with other $\beta$-blockers [aOR 2.10; CI 1.66$2.43] .{ }^{22}$ Similarly, the risk for preterm birth associated with labetalol [aOR 2.74; CI 2.393.13] was comparable to that associated with other $\beta$-blockers [aOR 1.69; CI 1.41-2.03]. ${ }^{22}$ Labetalol was associated with an increased risk of perinatal mortality [aOR 2.08 CI 1.263.44] whereas other $\beta$-blockers were not [aOR 1.71; CI 0.85-3.48]. ${ }^{22}$ Similar associations with SGA and preterm birth were confirmed with exposure to methyldopa and calcium channel blockers.

Women treated with antihypertensive agents in pregnancy are clearly at increased risk for adverse maternal outcomes, SGA and preterm birth. The indication for therapy,

hypertension, is clearly a risk factor that is associated with risk independent of treatment and may explain much of the associations attributed to treatment. Differential impact of different drugs is not consistent across studies and is confounded by the potential severity of hypertension across groups.

Abalos et al have recently completed a comprehensive review for The Cochrane Collaborative. ${ }^{24}$ The strongest finding of the analysis indicates that treatment of mild to moderate hypertension with any agent reduces the risk for severe hypertension [OR 0.50; CI 0.41-0.61]. ${ }^{24}$ Treatment with $\beta$-blockers is associated with a somewhat larger reduction in risk, [OR 0.38; CI 0.26-0.57]. ${ }^{24}$ While the reduction of risk is substantial with narrow confidence intervals, some have argued that the outcome "severe hypertension" is not clinically significant. Others have argued that the development of severe hypertension is a clear precursor to maternal morbidity and mortality and should be addressed.

The analysis suggests that treatment with anti-hypertensive agents, when assessed as a group, does not reduce the risk for the development of preeclampsia [OR 0.97; CI 0.83 1.13]. ${ }^{24}$ However when stratified by agent, treatment with a $\beta$-blocker is associated with reduced risk for preeclampsia [OR 0.73; CI 0.57-0.94], and treatment with calcium channel blockers is associated with an increased risk [OR 1.40; CI $1.06-1.86$ ]. ${ }^{24}$ The apparent differential effect by class of agent suggests an importance to the pharmacodynamic effects of classes of drugs as discussed above. A mechanism of action for the effect of $\beta$-blockers has been suggested by Carr et al. ${ }^{25}$ Treatment of women at risk for preeclampsia reduced the rise in sFlt-1 compared to that seen in low risk controls.

\section{Impact of Antihypertensive Therapy on Growth}

Fetal growth is clearly at risk in pregnancies complicated by hypertension. The hemodynamic characteristics of a woman's hypertension have been demonstrated to impact growth. In hypertensive women presenting prior to 28 weeks, those characterized by high resistance compared to those characterized by high cardiac output delivered babies weighing 
$1058 \mathrm{~g}$ less $(\mathrm{P}=0.001)$, delivered 4 weeks earlier, $(\mathrm{P}=0.001)$ and with a lower percentile birthweight, $\left(19^{\text {th }}\right.$ vs $\left.39^{\text {th }}, \mathrm{P}=0.005\right) .{ }^{26}$ High resistance represents a more advanced form of hypertension requiring earlier delivery and proportionately smaller babies.

The impact of treatment of hypertension on fetal growth has been the subject of significant concern. To address these concerns von Dadelszen et al have analyzed randomized trials with a technique of metaregression to not only examine for an effect but also the magnitude of effect. ${ }^{27}$ They reported that each fall in mean arterial pressure of $10 \mathrm{~mm} \mathrm{Hg}$ was associated with a $145 \mathrm{~g}$ decrease in birth weight. Differences in mean arterial pressure explained only $16 \%$ of the observed variation. The decrease in birth weight was not associated with the class of agent or duration of therapy. The analysis of Abalos confirms a risk for SGA associated with $\beta$-blockers [OR 1.38; CI 0.99-1.92] though with a confidence interval just crossing 1.0. ${ }^{24}$ In an unexpected finding, treatment with a $\beta$-blocker was associated with a reduction of risk for respiratory distress syndrome [OR 0.28; CI 0.110.71 ] a magnitude of impact comparable to antenatal steroids. ${ }^{24}$ Despite prior reports of associations with $\beta$-blockers and neonatal hypoglycemia, the analysis did not confirm an association [aOR 0.73; CI 0.24-2.24]. ${ }^{24}$

As summarized above, Lydakis et al reported that atenolol was associated with a lower birth weight, a trend to earlier delivery, and a trend to SGA compared to women treated with alternative monotherapy but comparable to women treated with dual therapy. ${ }^{23}$ This report has been largely responsible for concerns about atenolol in pregnancy. In contrast, Orbach reported that women with chronic hypertension treated with atenolol compared to women treated with methyldopa have a lower rate of preterm delivery (16.8\% vs $25.3 \%$ ), and a comparable rate of IUGR, $(8.4 \%$ vs $7.1 \%) .{ }^{21}$ They concluded that the observed adverse events seen among women treated for hypertension were due to the hypertension itself rather than to the choice of therapy. Although labetalol is commonly recommended for use to avoid the impact on the fetus, Meidahl et al reported comparable risks of SGA for infants exposed to labetalol [2.02; CI 1.71-2.37] compared to those exposed to other $\beta$-blockers [OR 2.10; CI 1.66-2.43], to methyldopa [OR 1.43; CI 1.04-1.96], or to calcium channel blockers [OR 1.88; 1.02-3.49]. ${ }^{22}$ An increased risk for stillbirth [OR 2.08; CI 1.26-3.44] was found for infants exposed to labetalol but not for other antihypertensive medications. ${ }^{22}$ Retrospective data analysis of hypertensive drug exposure is, by its very nature, confounded by hypertension itself and the severity of hypertension that frequently cannot be assessed. The data to date confirm that hypertension is clearly associated with an impact on fetal growth. The suggestion that some agents are of greater risk than others is not generally supported. Data from von Dadelszen's analysis does suggest that treatment may have a price, but a small price, $145 \mathrm{~g}$ per $10 \mathrm{mmHg}$ of reduction in mean arterial pressure. ${ }^{27}$ The balance of maternal benefits must be weighed against this reduction in growth potential.

Some insight into mechanism of reduction in fetal growth can be ascertained from investigations where maternal hemodynamic measurements were available to guide and assess the impact of therapy. In a randomized trial where atenolol was used to treat prehypertension in hyperdynamic women, those treated with atenolol delivered babies smaller than those not treated, $(\mathrm{P}=0.02)$ but not smaller than women at low risk for the development of preeclampsia. ${ }^{28}$ The rates of SGA in the groups were comparable, atenolol 
4.7\% compared to placebo $5.2 \% .{ }^{28}$ In part, the differences in mean birth weight were associated with less macrosomia in the atenolol group. When maternal hemodynamic responses were evaluated, the smallest 4 infants in the atenolol group were associated with a maternal cardiac output that fell the mean for gestational age. ${ }^{28}$ In a subsequent study of women at risk for preeclampsia who had a high cardiac output treated with atenolol, a cardiac output that fell below the mean for gestational age or a vascular resistance that rose above 1150 dyne $\bullet \mathrm{cm} \bullet \mathrm{sec}^{-4}$, were strongly associated with a risk for low birth weight percentile, $(\mathrm{P}=0.001) .{ }^{29}$ Clonidine is an antihypertensive with different mechanisms of action from $\beta$-blockers. In some women, as described above, the primary hemodynamic action is a reduction in heart rate and cardiac output. In these women who had increased vascular resistance prior to therapy, a reduction in cardiac output was associated with a higher rate of IUGR compared to women who experienced vasodilation, (41\% vs. $8.8 \%$, $\mathrm{P}=0.008) .{ }^{17}$ These results suggest that maternal hemodynamic conditions are important to fetal growth probably though their effects on uteroplacental blood flow. Baseline conditions of reduced perfusion, low cardiac output and high resistance, are associated with reduced growth. Treatment that results in these conditions, independent of the agent, seems also to result in reduced growth potential.

Table 2 summarizes results on maternal and fetal outcomes. Insufficient data has led to no strong recommendation for therapy. The data that do exist suggest potential benefits associated with treatment: reduction in severe hypertension, reduction in the risk of preeclampsia, and reduction in the rate of respiratory distress syndrome. They also suggestions a risk of IUGR. There are suggestions that benefits and risks may be associated with the specificity of drugs used. A dose effect is present in the pharmacological treatment of hypertension. There is little information regarding dose effect on potential complications such as fetal growth though, from a mechanistic point of view, it would seem likely. While short-term fetal and neonatal growth data have been evaluated, no information is available on the long-term outcomes of children born to women who were treated or not treated for their hypertension. To answer these questions there is compelling need to conduct a body of research comparable to that which informs the treatment of hypertension in non-pregnant individuals. Until that is available, clinicians must make management decisions based on interpretation the existing data and their clinical judgment. To design appropriate research and to treat pregnant women, an understanding of the changes in drug disposition and drug effects on the mother and fetus is required.

\section{Summary}

The pharmacological management of hypertension in pregnancy remains controversial and understudied. Pregnancy is associated with changes in the clearance of most antihypertensive agents. These changes result in the need to consider modifications in dosage and dosing interval. In some cases, increased variability across patients requires individualization of dosing based on clinical response. Pregnancy and the hypertensive conditions of pregnancy are associated with changes in baseline hemodynamic conditions that may impact the choice of pharmacological agent. A balance of pharmacodynamics effects need to be achieved to benefit mother and fetus. The outcomes of pregnancies where hypertension is treated clearly need investigation to inform appropriate care. 
Individualization of care based on specific characteristics of a pregnancy and the pharmacokinetics and pharmacodynamics of specific drugs will likely be necessary.

\section{Acknowledgments}

National Institutes of Health Eunice Kennedy Shriver National Institute of Child Health and Human Development [Grant U10- HD047892] (in support of the Obstetric-Fetal Pharmacology Research Unit Network).

\section{References}

1. Chobanian AV, Bakris GL, Black HR, et al. Seventh report of the Joint National Committee on Prevention, Detection, Evaluation, and Treatment of High Blood Pressure. Hypertension. 2003; 42(6):1206-52. [PubMed: 14656957]

2. The Joint Commission: "Preventing maternal death,". [Accessed February 21, 2014] Sentinel Event Aleart. Jan 26. 2010 http://www.jointcommission.org/assets/1/18/sea_44.pdf

3. Hypertension in Pregnancy Report of the American College of Obstetricians and Gynecologists' Task Force on Hypertension in Pregnancy. Obstet Gynecol. 2013; 122(5):1122-31. [PubMed: 24150027]

4. Hebert MF, Carr DB, Anderson GD, et al. Pharmacokinetics and pharmacodynamics of atenolol during pregnancy and postpartum. J Clin Pharmacol. 2005; 45(1):25-33. [PubMed: 15601802]

5. Zanger UM, Schwab M. Cytochrome P450 enzymes in drug metabolism: regulation of gene expression, enzyme activities, and impact of genetic variation. Pharmacol Ther. 2013; 138(1):10341. [PubMed: 23333322]

6. Wadelius M, Darj E, Frenne G, Rane A. Induction of CYP2D6 in pregnancy. Clin Pharmacol Ther. 1997; 62(4):400-7. [PubMed: 9357391]

7. Högstedt S, Rane A. Plasma concentration-effect relationship of metoprolol during and after pregnancy. Eur J Clin Pharmacol. 1993; 44(3):243-6. [PubMed: 8491238]

8. Rogers RC, Sibai BM, Whybrew WD. Labetalol pharmacokinetics in pregnancy-induced hypertension. Am J Obstet Gynecol. 1990; 162(2):362-6. [PubMed: 2309815]

9. Influence of gestational diabetes mellitus on the stereoselective kinetic disposition and metabolism of labetalol in hypertensive patients. Eur J Clin Pharmacol. 2011; 67(1):55-61. [PubMed: 20848091]

10. Buchanan ML, Easterling TR, Carr DB, et al. Clonidine pharmacokinetics in pregnancy. Drug Metab Dispos. 2009; 37(4):702-5. [PubMed: 19116263]

11. Claessens AJ, Risler LJ, Eyal S, Shen DD, Easterling TR, Hebert MF. CYP2D6 mediates 4hydroxylation of clonidine in vitro: implication for pregnancy-induced changes in clonidine clearance. Drug Metab Dispos. 2010; 38(9):1393-6. [PubMed: 20570945]

12. Hebert MF, Easterling TR, Kirby B, et al. Effects of pregnancy on CYP3A and P-glycoprotein activities as measured by disposition of midazolam and digoxin: a University of Washington specialized center of research study. Clin Pharmacol Ther. 2008; 84(2):248-53. [PubMed: 18288078]

13. Prevost RR, Akl SA, Whybrew WD, Sibai BM. Oral nifedipine pharmacokinetics in pregnancyinduced hypertension. Pharmacotherapy. 1992; 12(3):174-7. [PubMed: 1608848]

14. Barton JR, Prevost RR, Wilson DA, Whybrew WD, Sibai BM. Nifedipine pharmacokinetics and pharmacodynamics during the immediate postpartum period in patients with preeclampsia. Am J Obstet Gynecol. 1991; 165(4 Pt 1):951-4. [PubMed: 1951561]

15. Haas DM, Quinney SK, Clay JM, et al. Nifedipine pharmacokinetics are influenced by CYP3A5 genotype when used as a preterm labor tocolytic. Am J Perinatol. 2013; 30(4):275-81. [PubMed: 22875663]

16. Easterling TR, Benedetti TJ, Schmucker BC, Carlson KL. Antihypertensive therapy in pregnancy directed by noninvasive hemodynamic monitoring. Am J Perinatol. 1989; 6(1):86-9. [PubMed: 2910324] 
17. Rothberger S, Carr D, Brateng D, Hebert M, Easterling TR. Pharmacodynamics of clonidine therapy in pregnancy: a heterogeneous maternal response impacts fetal growth. Am J Hypertens. 2010; 23(11):1234-40. [PubMed: 20725050]

18. Carr DB, Gavrila D, Brateng D, Easterling TR. Maternal hemodynamic changes associated with furosemide treatment. Hypertens Pregnancy. 2007; 26(2):173-8. [PubMed: 17469007]

19. Easterling TR, Benedetti TJ, Schmucker BC, Millard SP. Maternal hemodynamics in normal and preeclamptic pregnancies: a longitudinal study. Obstet Gynecol. 1990; 76(6):1061-9. [PubMed: 2234714]

20. Bosio PM, McKenna PJ, Conroy R, O’Herlihy C. Maternal central hemodynamics in hypertensive disorders of pregnancy. Obstet Gynecol. 1999; 94(6):978-84. [PubMed: 10576186]

21. Orbach H, Matok I, Gorodischer R, et al. Hypertension and antihypertensive drugs in pregnancy and perinatal outcomes. Am J Obstet Gynecol. 2013; 208(4):301, e1-6. [PubMed: 23159698]

22. Meidahlpetersen K, Jimenez-solem E, Andersen JT, et al. $\beta$-Blocker treatment during pregnancy and adverse pregnancy outcomes: a nationwide population-based cohort study. BMJ Open. 2012; 2(4)

23. Lydakis C, Lip GY, Beevers M, Beevers DG. Atenolol and fetal growth in pregnancies complicated by hypertension. Am J Hypertens. 1999; 12(6):541-7. [PubMed: 10371362]

24. Abalos E, Duley L, Steyn DW. Antihypertensive drug therapy for mild to moderate hypertension during pregnancy. Cochrane Database Syst Rev. 2014; 2:CD002252. [PubMed: 24504933]

25. Carr DB, Tran LT, Brateng DA, et al. Hemodynamically-directed atenolol therapy is associated with a blunted rise in maternal sFLT-1 levels during pregnancy. Hypertens Pregnancy. 2009; 28(1):42-55. [PubMed: 19165669]

26. Easterling TR, Benedetti TJ, Carlson KC, Brateng DA, Wilson J, Schmucker BS. The effect of maternal hemodynamics on fetal growth in hypertensive pregnancies. Am J Obstet Gynecol. 1991; 165(4 Pt 1):902-6. [PubMed: 1951551]

27. Von dadelszen P, Ornstein MP, Bull SB, Logan AG, Koren G, Magee LA. Fall in mean arterial pressure and fetal growth restriction in pregnancy hypertension: a meta-analysis. Lancet. 2000; 355(9198):87-92. [PubMed: 10675164]

28. Easterling TR, Brateng D, Schmucker B, Brown Z, Millard SP. Prevention of preeclampsia: a randomized trial of atenolol in hyperdynamic patients before onset of hypertension. Obstet Gynecol. 1999; 93(5 Pt 1):725-33. [PubMed: 10912975]

29. Easterling TR, Carr DB, Brateng D, Diederichs C, Schmucker B. Treatment of hypertension in pregnancy: effect of atenolol on maternal disease, preterm delivery, and fetal growth. Obstet Gynecol. 2001; 98(3):427-33. [PubMed: 11530124] 


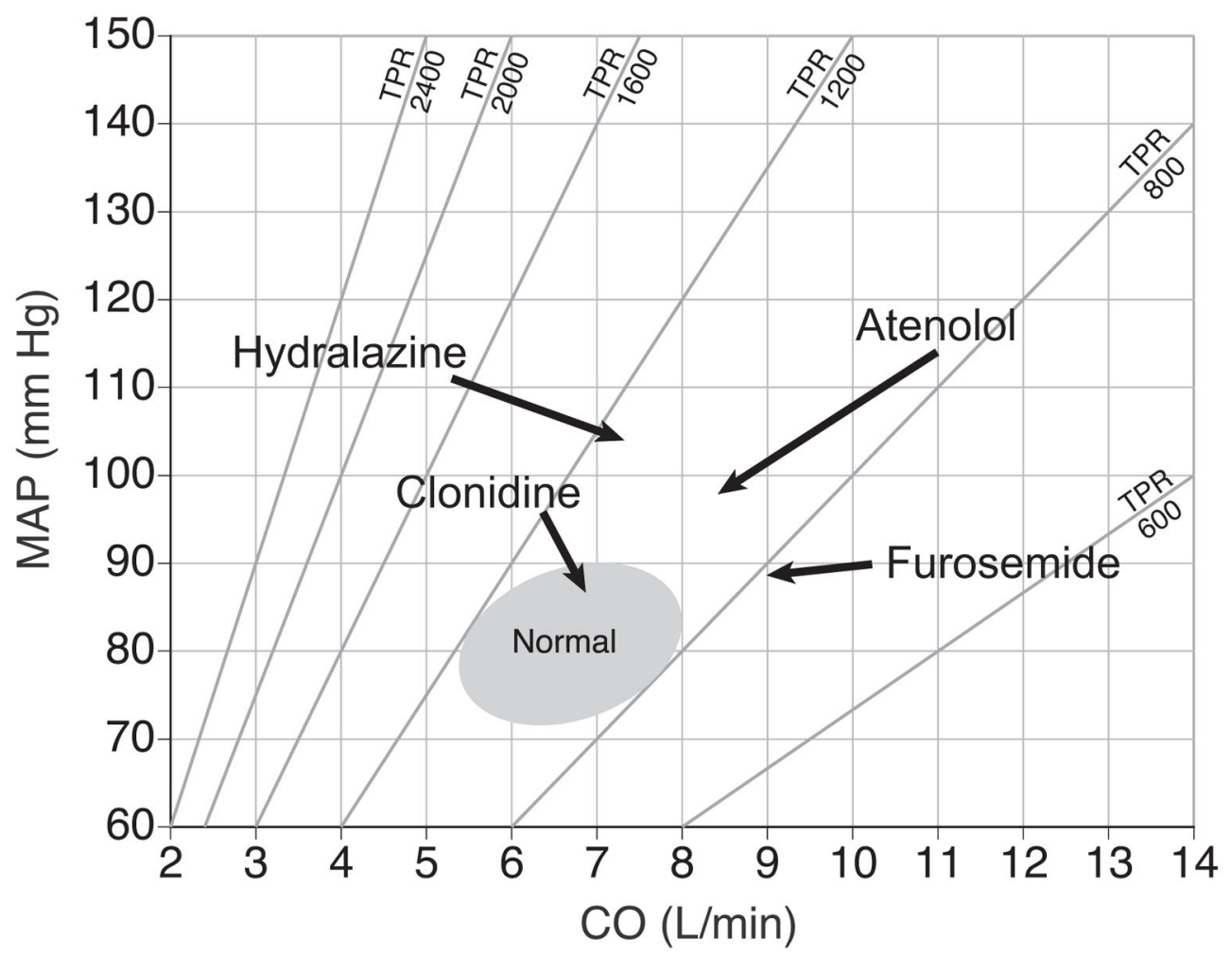

Figure 1. Hemodynamic Effects of Antihypertensive Agents

Cardiac output is plotted vs. mean arterial pressure with isometric diagonal lines of vascular resistance to graphically represent Ohm's Law: MAP $=(\mathrm{TPR} / 80) \cdot \mathrm{CO}$. Hemodynamic vectors of change resulting from vasodilation will run perpendicular to lines of resistance. Vectors of change due to a change in cardiac output with no change in resistance will run parallel to lines of resistance. The average hemodynamic response to individual drugs in pregnancy is represented by labeled vectors. 


\section{Table 2}

\section{Summary of Outcome Studies}

\begin{tabular}{|c|c|c|c|}
\hline Outcome & Comparison & Finding & $\underline{\text { Source }}$ \\
\hline \multirow[t]{3}{*}{ Severe Htn } & Mild-Mod Htn: Rx vs None & & \\
\hline & Any drug & [OR 0.50; CI 0.41-0.61] & Meta-A $\mathrm{A}^{24}$ \\
\hline & $\beta$-blocker & [OR 0.38; CI 0.26-0.57] & Meta-A $\mathrm{A}^{24}$ \\
\hline \multirow[t]{5}{*}{ Preeclampsia } & Mild-Mod Htn: Rx vs None & & \\
\hline & Any drug & [OR 0.97; CI $0.83-1.13$ ] & Meta-A $\mathrm{A}^{24}$ \\
\hline & $\beta$-blocker & [OR $0.73 ;$ CI $0.57-0.94]$ & Meta-A $\mathrm{A}^{24}$ \\
\hline & Ca channel blocker & [OR $1.40 ;$ CI $1.06-1.86]$ & Meta-A $\mathrm{A}^{24}$ \\
\hline & Methyldopa vs atenolol & $(27 \%$ vs $17 \%)$ & $\mathrm{AdminDB}^{21}$ \\
\hline \multirow[t]{7}{*}{ SGA } & All $\beta$-blocker vs no Htn & [aOR 1.98; CI 1.75-2.23] & $\operatorname{AdminDB}^{22}$ \\
\hline & Pure $\beta$-blocker vs no Htn & [aOR 2.10; CI 1.66-2.43] & $\operatorname{AdminDB}^{22}$ \\
\hline & Labetalol vs no Htn & [aOR 2.12; 1.72-2.37] & $\operatorname{AdminDB}^{22}$ \\
\hline & $\beta$-blocker vs no $\mathrm{Rx}$ & [OR 1.38; CI 0.99-1.92] & Meta-A ${ }^{24}$ \\
\hline & $\beta$-blocker vs no Rx & $4.7 \%$ vs $5.2 \%$ & $\mathrm{RCT}^{28}$ \\
\hline & Mult drug vs Atenolol vs other single drug vs no Rx & $46 \%$ vs. $33 \%$ vs $26 \%$ vs $21 \%$ & $\operatorname{AdminDB}^{23}$ \\
\hline & Methyldopa vs atenolol & $2.4 \%$ vs $5.6 \%$ & $\operatorname{AdminDB}^{21}$ \\
\hline \multirow[t]{2}{*}{ IUGR } & anti-HTN vs no Htn & [OR 4.37; CI 3.00-6.36] & $\operatorname{AdminDB}^{21}$ \\
\hline & Methyldopa vs atenolol & 4.32 vs $5.19 \%$ & $\operatorname{AdminDB}^{21}$ \\
\hline \multirow[t]{4}{*}{ Preterm Delivery } & anti-HTN vs no Htn & [OR 3.69; CI 2.90-4.69] & AdminDB $^{21}$ \\
\hline & Methyldopa vs atenolol & $17 \%$ vs $9 \%$ & $\mathrm{AdminDB}^{21}$ \\
\hline & Pure $\beta$-blocker vs no Htn & [aOR 1.69; CI 1.41-2.03]. & $\operatorname{AdminDB}^{22}$ \\
\hline & Labetalol vs no Htn & [aOR 2.74; CI 2.39-3.13] & $\operatorname{AdminDB}^{22}$ \\
\hline \multirow[t]{3}{*}{ Perinatal Mortality } & All $\beta$-blocker vs no Htn & [aOR 1.89; CI 1.25-2.84] & $\operatorname{AdminDB}^{22}$ \\
\hline & Pure $\beta$-blocker vs no Htn & [aOR 1.71; CI 0.85-3.48] & $\operatorname{AdminDB}^{22}$ \\
\hline & Labetalol vs no Htn & [aOR 2.08 CI 1.26-3.44] & $\operatorname{AdminDB}^{22}$ \\
\hline RDS & $\beta$-blocker vs no Rx & [OR 0.28; CI 0.11-0.71] & Meta-A ${ }^{24}$ \\
\hline Hypoglycemia & $\beta$-blocker vs no $\mathrm{Rx}$ & [aOR 0.73; CI 0.24-2.24] & Meta-A $\mathrm{A}^{24}$ \\
\hline
\end{tabular}

Htn - hypertension; Rx - treatment; Mult - multiple; SGA - small for gestational age

IUGR - intrauterine growth restriction; RDS - respiratory distress syndrome;

Meta-A - meta analysis; AdminBD - administrative data base; RCT - randomized controlled trial 